\title{
Impact of Thermal Annealing under Nitrogen Ambient on Structural, Micro-Raman, and Thermogravimetric Analyses of Camphoric-CNT
}

\author{
M. S. Shamsudin, ${ }^{1,2}$ A. B. Suriani, ${ }^{3}$ S. Abdullah, ${ }^{2}$ S. Y. S. Yahya, ${ }^{2}$ and M. Rusop ${ }^{1,4}$ \\ ${ }^{1}$ Centre of Nanoscience and Nanotechnology (NANO-SciTech Centre), Institute of Science, Universiti Teknologi MARA, Selangor, \\ 40450 Shah Alam, Malaysia \\ ${ }^{2}$ School of Physics and Material Studies, Faculty of Applied Sciences, Universiti Teknologi MARA, Selangor, \\ 40450 Shah Alam, Malaysia \\ ${ }^{3}$ Department of Physics, Faculty of Sciences and Mathematics, Universiti Pendidikan Sultan Idris, Perak, \\ 35900 Tanjung Malim, Malaysia \\ ${ }^{4}$ NANO-ElecTronic Centre, Faculty of Electrical Engineering, Universiti Teknologi MARA, Selangor, 40450 Shah Alam, Malaysia
}

Correspondence should be addressed to M. S. Shamsudin; nanopizza@rocketmail.com

Received 23 June 2012; Accepted 3 August 2012

Academic Editor: Roberto Flammini

Copyright (c) 2013 M. S. Shamsudin et al. This is an open access article distributed under the Creative Commons Attribution License, which permits unrestricted use, distribution, and reproduction in any medium, provided the original work is properly cited.

A systematical study of the effects on thermal annealing treatment under nitrogen ambient on the structural, micro-Raman, and thermogravimetric analyses of camphoric-carbon nanotubes (CNT) has been undertaken. Heat treatment of camphoric-CNT under nitrogen ambient was found to be an efficient technique of removing noncrystalline carbon and residual transition metal. Based on structural analysis, the heat-treated samples showed a clear view of overall camphoric-CNT structure.

\section{Introduction}

Over the last two decades, the official birth date of carbon nanotubes (CNT) was attributed to Japanese microscopist named Sumio Iijima. He was considered a "father of CNT" at that time affiliated at NEC Corporation, Japan, perished in a paper which shows his effort on the first discovery and remarkable potential of a new class of nano-structured carbon material [1]. It is important to notice that a common type of CNT is single-walled CNT (SWCNT) [2] and multiwalled CNT (MWCNT) [3] which can be visualized as a single and concentrically rolled-up sheet/s of graphene, respectively. CNT has gained great interests due to its superior intrinsic properties in terms of high thermal conductivity $[4,5]$, excellent mechanical strength $[6,7]$, and effective field emission characteristics $[8,9]$ suggesting their applicability for energy storage and conversion [10], filler in polymer nanocomposites [11], and flat-panel display [12]. There are several methods to eliminate the impurities in the as-synthesized
CNT, including microwave digestion and radiation [13], reflux in acidic medium [14], and ultrasonication [15].

In the present paper, among the various purification techniques, we adopted thermal annealing treatment under nitrogen ambient coupled with different thermal annealing time by single-stage catalytic chemical vapor deposition (CVD) method. The effect of thermal annealing treatment time under nitrogen ambient on structural, micro-Raman, and thermogravimetric analysis was investigated. It demonstrated that nonoxidative gas-phase purification involves the elimination processes of some organometallic and carbonaceous particles from nanotubes. However, few effective methods have been reported for the removal of as-synthesized impurities, such as amorphous carbon (a-C) and catalyst without damaging the camphoric-CNT structure. Finally, this finding describes the removal-mechanism in terms of the strong relation between non-oxidative gas seniority upon exposure to form heat-treated camphoric-CNT was sought. 


\section{Experimental Description}

2.1. Sample Preparation. In this study, camphoric-CNT was grown using two-stage catalytic CVD apparatus [16]. The processing and experimental setup/apparatus of assynthesized camphoric-CNT were described elsewhere [17]. The optimized condition of $10 \% \mathrm{w} / \mathrm{v}$ (weight-volume percentage) of ferrocene relative to camphor oil was placed in different alumina boats. The alumina boats were positioned side by side along the quartz tube in the first furnace of two-stage catalytic CVD apparatus. The pretreatment of the evaporated camphor oil and sublimated ferrocene occur at $180^{\circ} \mathrm{C}$ at first furnace. The growth phase lasted for one hour at $800^{\circ} \mathrm{C}$ [18]. After the completion of growth phase, the first and second furnaces were shut down until room temperature was reached under constant cooling rate and then was ready for thermal annealing process.

The synthesized camphoric-CNT was then transferred to the thermal annealing system as can be seen in Figure 1. At first, each time, $0.5 \mathrm{~g}$ of synthesized camphoric-CNT was loaded into the system. The thermal annealing time was determined to fix the thermal annealing temperature. During the thermal annealing experiment, the system was kept at about $450^{\circ} \mathrm{C}$ in a lower flow rate of nitrogen $(\sim 10 \mathrm{sccm})$ and the thermal annealing times were changed from 2 to 10 minutes with 2-minute interval. Finally, at the same time, the mechanism of the thermal annealing process of each camphoric-CNT was discussed. In order to compensate such a drawback, as the time proceeds, it is hoped that more camphoric-CNT were exposed to the graphitic layer and have more chances to be attacked by the nitrogen molecules.

2.2. Characterization Techniques. The surface morphology and structural behaviors of as-synthesized and annealed camphoric-CNT were characterized comprehensively using a field emission scanning electron microscope (FESEM, JEOL JSM-7600F) operated at $10 \mathrm{kV}$ electron high tension (EHT) and $200 \mathrm{kX}$ magnification. The degree of defect and graphitization of each sample was observed by a microRaman spectrometer ( $\mu$-Raman, HORIBA JOBIN HR800 Lab-Ram System) equipped with a $514.532 \mathrm{~nm}$ wavelengths $\mathrm{Ar}^{+}$laser excitation as the light source. The power of the laser was adjusted by optical filter with a $100 \mathrm{X}$ objective lens. The $\mu$-Raman spectra were recorded from 100 to $2000 \mathrm{~cm}^{-1}$. The thermogravimetric analysis was performed by thermogravimetric analyzer (TG, Perkin Elmer Pyris 1 TGA), operating at temperatures range from room temperature to $1000^{\circ} \mathrm{C}$ at a heating rate of $20^{\circ} \mathrm{C} / \mathrm{min}$ in nitrogen ambient. The yield of camphoric-CNT before and after thermal annealing was determined.

\section{Result and Discussion}

3.1. Surface Morphology. The FESEM micrographs which analyzed the variation of as-synthesized and annealed $(2,4,6$, 8 , and 10 minutes thermal annealing procedure) camphoricCNT surface structures were shown in the Figure 2. In addition, the size of camphoric-CNT was determined and compared before and after a heat treatment process. From
Figure 2(a), it is clear that as-synthesized camphoric-CNT has large-diameter ranges from 49.1 to $57.3 \mathrm{~nm}$. The CNT diameter was slightly decreased to $51.4 \mathrm{~nm}$ as the thermal annealing was for 2 minutes as in Figure 2(b). As the time annealing increases further (from 4 to 6 minutes) the tube's diameter was further decreased to smaller diameter range (26.7 to $22.1 \mathrm{~nm})$. However, further increase in thermal annealing time (from 8 to 10 minutes) did not show any significant changes in tube's diameter.

The modification of the surface structure towards smoother morphology was also confirmed. Figure 2(a) showed the originality of camphoric-CNT, where the surface structure looks rough, uneven with bulge-like structure. These were believed due to a-C which was deposited on the tube wall. However, after the thermal annealing procedures were done, the reductions of tube's diameter were clearly observed. Moreover, rough and uneven surfaces with a bulge still remain as shown in Figure 2(b). This shows that within 2-minute times of thermal annealing, procedures were not sufficient to eliminate the impurities that produced during the synthesis process. This suggested that all contaminant compounds and other impurities (i.e., a-C and byproduct carbon) were completely burnt off at 6-minute times without any camphoric-CNT structure collapse. We found that the smallest tube's diameter was obtained at 6 minutes of thermal annealing time. However, the smoother and impurities-free surface was spotted at annealing time as shown in Figures 2(d) and 2(f), respectively. This study emphatically proves that by reordering the carbon structure, the sample is almost free from a-C layer in which tube wall produces relatively hollow and bright inside. As confirmed by FESEM micrograph as in Figure 2(f), the dense camphoric-CNT is completely transformed to translucent camphoric-CNT (marked with red circle). It is found that when they overlapped, we could see another transparent (see-through) CNT-2 behind CNT-1.

We are not matured enough to understand the principle of a removal mechanism of the world inside nanotubes unless by providing specialized laboratory equipment, we will be able to do insitu measurement. There are many basic issues and highly debatable questions concerning the removal mechanism awaiting solid answers to fulfill expectations [19]. However, it is hard to visualize the crucial role of nitrogen atmosphere in removal-mechanism of camphoric-CNT. It is important to note that under these theoretical assumptions and experimental verifications, is discussed below:

(i) To elucidate this, consider the relationship of kinetic energy and motion in gas phase. At a lower temperature $\left(-273.15^{\circ} \mathrm{C}\right)$, the particles of nitrogen (constituent of molecules) have no kinetic energy, in which the molecules are as closely as possible in complete rest, and it defines two things: (a) nitrogen molecules have minimal motion and (b) they maintain only quantum-mechanical motion [20]. Though nitrogen is considered as nearly inert gas. However, at higher temperature $\left(\sim 450^{\circ} \mathrm{C}\right)$, nitrogen molecules can react with carbon material as thermal annealing treatment time increased. It seems more reasonable to associate the thermal annealing treatment to 


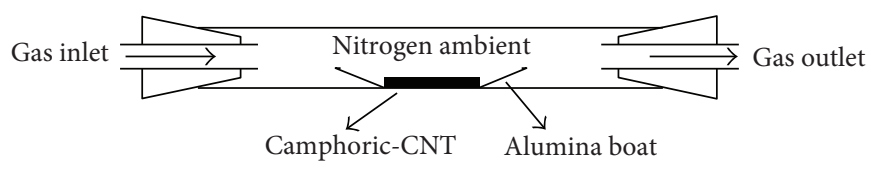

FIGURE 1: Schematic diagram of the apparatus that used for thermal annealing system.

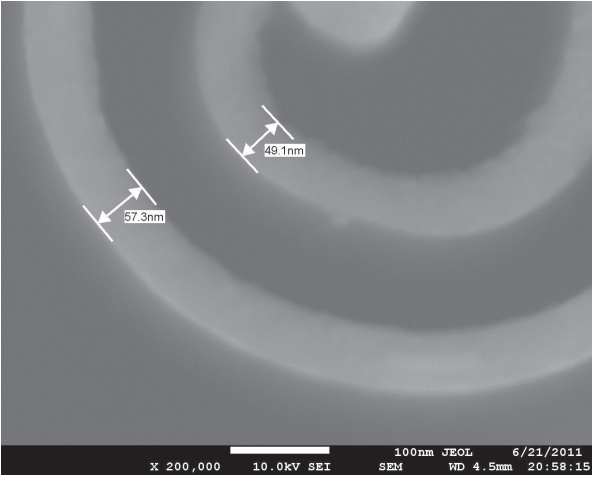

(a)

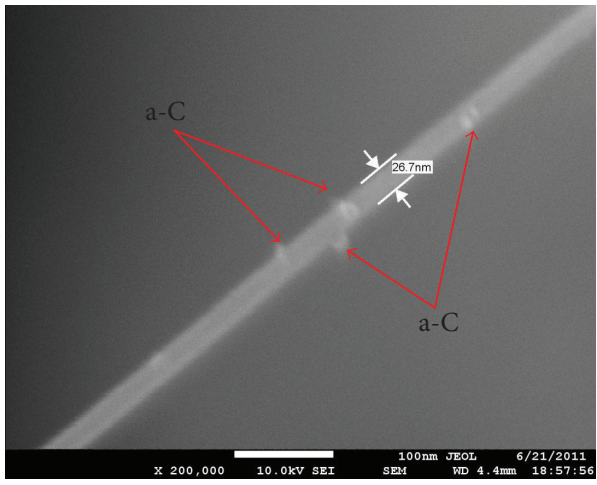

(c)

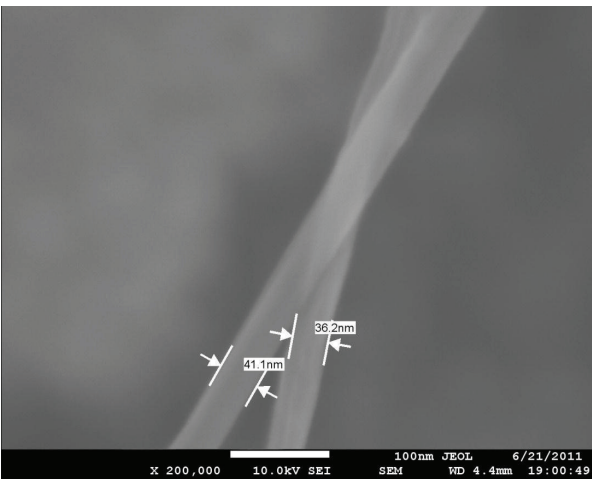

(e)

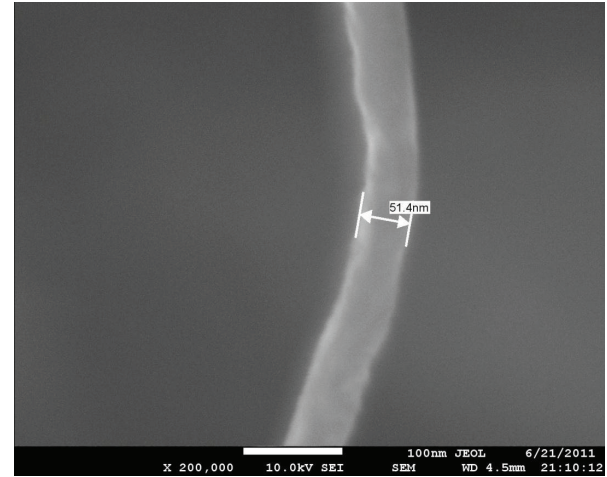

(b)

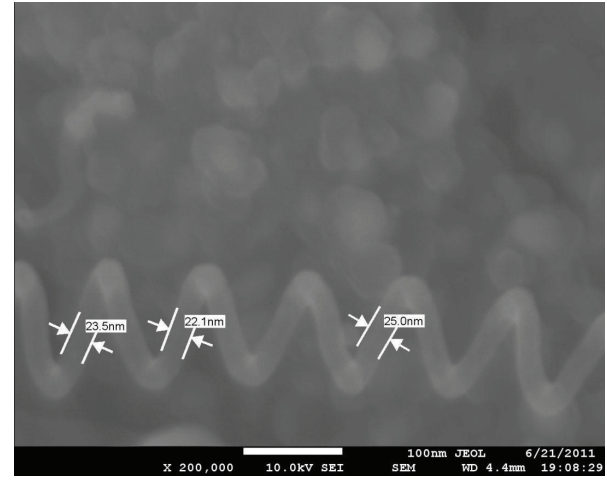

(d)

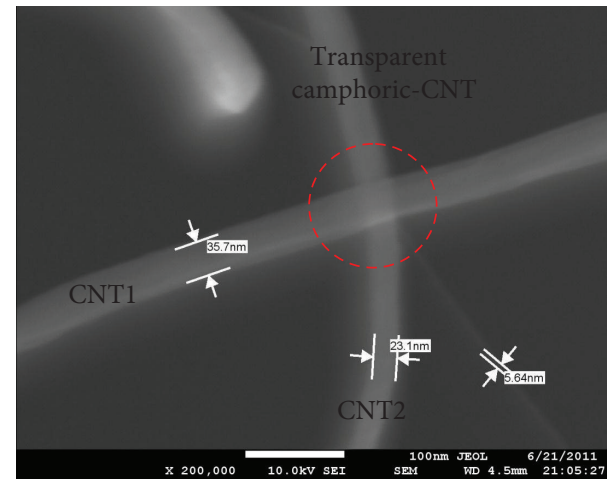

(f)

Figure 2: Typical FESEM images of the camphoric-CNT annealed at different thermal annealing time with $450^{\circ} \mathrm{C}$ thermal annealing temperature: (a) as-synthesized, (b) 2, (c) 4, (d) 6, (e) 8, and (f) 10 minutes.

the nature of kinetic energy nitrogen gas. It is a certain kind of vibrational motion of its constituent nitrogen molecules called translational motion in gas. As expected, nitrogen molecules move in threedimensional space $(x-, y$-, and $z$-axis). It means that the nitrogen molecules move in spatial degrees of freedom. We believe that a-C layer were placed and stuck together on the outer layer of camphoric-CNT. There are several points of support in the abovementioned removal mechanism. It is speculated that 


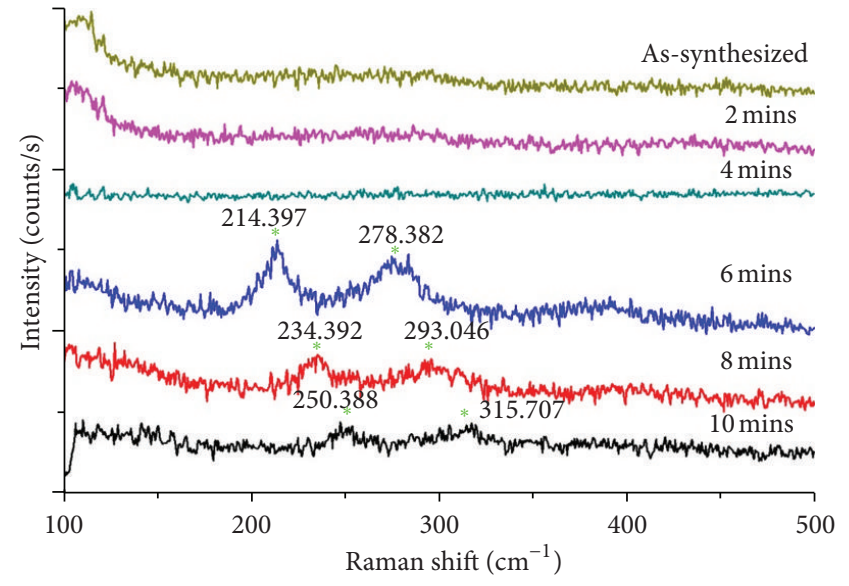

(a)

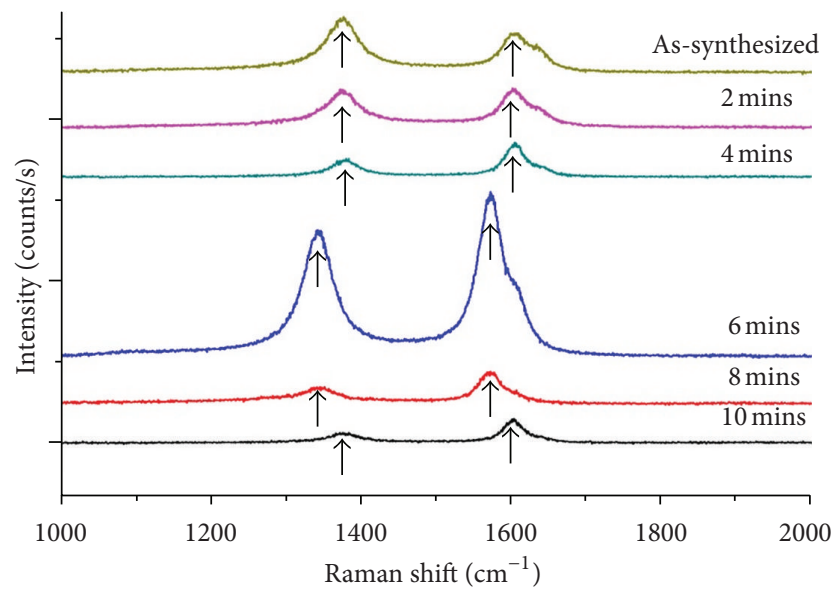

(b)

FIGURE 3: Micro-Raman spectra of camphoric-CNT grown by two-stage catalytic CVD at different thermal annealing time for the following: (a) RBM and (b) TM. Positions of $\omega_{\mathrm{RBM}}\left(^{*}\right)$ and $\omega_{\mathrm{TM}}$ peaks are shown.

nitrogen molecules behave as a oxidizing agent and a-C will be etched layer by layer by nitrogen molecules as thermal annealing treatment time increased.

(ii) Besides, nitrogen molecules act as a carrier agent. Since a-C layer has low thermal stability at a higher temperature compared to perfect ideal CNT [21]. This phenomenon could happen due to a-C layer sublimates at $450^{\circ} \mathrm{C}$. After that, a-C layer can be directly purged out from the single-stage thermal annealing system by nitrogen gas.

3.2. Micro-Raman Analysis. The degree of graphitization and crystal camphoric-CNT structure can be analyzed by microRaman spectroscopy at room temperature. Figures 3(a) and 3(b) show the micro-Raman spectra of as-synthesized and annealed camphoric-CNT using a $514.532 \mathrm{~nm}$ wavelengths $\mathrm{Ar}^{+}$laser excitation in the wavenumber range from 100 to $500 \mathrm{~cm}^{-1}$ and 1000 to $2000 \mathrm{~cm}^{-1}[22,23]$, respectively. Figure 3(a) shows that the normalized radial breath mode $(\mathrm{RBM})$ at the lower frequency region of small tubes is sensitive to the tube diameter $\left(d_{\text {SWCNT }}\right)$ and valid in the range of $0.5<d_{\text {SWCNT }}<2.48 \mathrm{~nm}$. By using Bandon's equation [24], the diameter of SWCNT can be evaluated from normalized intensity of RBM peak's position $\left(\omega_{\text {SWCNT }}\right)$, as $d_{\text {SWCNT }}=$ $248\left(\mathrm{nmcm}^{-1}\right) / \omega_{\text {SWCNT }}\left(\mathrm{cm}^{-1}\right)$. While for large tubes, the effect of tube diameter on the normalized RBM frequency is ignorable, this might be due to the existence of MWCNT. As shown in Figure 3(b), we can see the deconvolution spectrum of tangential mode (TM) located at (i) $1380 \mathrm{~cm}^{-1}$ assigned to the disorder-induced phonon mode (D-band) and (ii) $1600 \mathrm{~cm}^{-1}$ designated $\mathrm{C}-\mathrm{C}$ stretching graphitic mode (G-band) [25]. In particular interest, the narrowing of the bands is the evaluation of the $I_{D} / I_{G}$ ratio. The lower $I_{D} / I_{G}$ ratio corresponds for better crystallinity of graphitic structure [26]. The improvement of the intensity ratio is proven by the appearance of defects in the as-synthesized camphoricCNT which decreased due to defect reduction process as the thermal annealing treatment time increased.

According to this study, the effect of thermal annealing treatment time under nitrogen ambient can be interpreted by using micro-Raman analysis. At the band area of RBM, no peaks related to SWCNT were observed for as-synthesized, 2and 4-minute treated camphoric-CNT samples. The peaks of SWCNT appear at 6-, 8-, and 10-minute treated camphoricCNT. The positions of $\omega_{\text {SWCNT }}$ were located at 214.4 and $278.4 \mathrm{~cm}^{-1}$ for 6 minutes, 234.4 and $293.0 \mathrm{~cm}^{-1}$ for 8 minutes, and 250.4 and $315.7 \mathrm{~cm}^{-1}$ for 10 minutes. From microRaman analysis, the calculated values of $d_{\text {SWCNT }}$ were summarized as in Table 1. The number of walls and diameter in MWCNT can be estimated by an empirical law and quickly determined using FESEM, if the nanotubes are individually distinguishable, which is suggested by Chiodarelli et al. [27]. There are many conflicting reports since the perfect ideal CNT is far from the practically producing CNT today. However, this theory is valid if the CNT is free from any contaminants (i.e., metal catalyst and a-C layer) and structural defect.

As shown in Figure 3(b) and Table 1, there is a gradual trend of decreas $I_{D} / I_{G}$ ratio as thermal annealing treatment time increased. Moreover, it is confirmed from the microRaman analysis that the lowest $I_{D} / I_{G}$ ratio is 0.442 at 10 minutes ofthermal annealing treatment time. Thus, the thermal annealing treatment time under nitrogen ambient has efficiently enhanced the removal of the structural defects.

3.3. Thermogravimetric Analysis. Normally, TG analysis is a part of quantitative determination to define the purity of CNT. In this study, the TG analysis was performed in the oxygen ambient, while the temperature increases at rate of $20^{\circ} \mathrm{C} / \mathrm{min}$. For every sample, TG analysis was done up 
TABLE 1: Tabulated data of $d_{\text {SWCNT }}$ and $I_{D} / I_{G}$ ratio for as-synthesized and at different thermal annealing treatment time using microRaman analysis.

\begin{tabular}{lcc}
\hline $\begin{array}{l}\text { Thermal annealing treatment time } \\
\text { (minutes) }\end{array}$ & $\begin{array}{c}d_{\text {SWCNT }} \\
(\mathrm{nm})\end{array}$ & $\begin{array}{c}I_{D} / I_{G} \\
\text { ratio }\end{array}$ \\
\hline As-synthesized & - & 1.365 \\
2 & - & 0.994 \\
4 & - & 0.878 \\
6 & $1.156,0.891$ & 0.799 \\
8 & $1.058,0.846$ & 0.575 \\
10 & $0.990,0.786$ & 0.442 \\
\hline
\end{tabular}

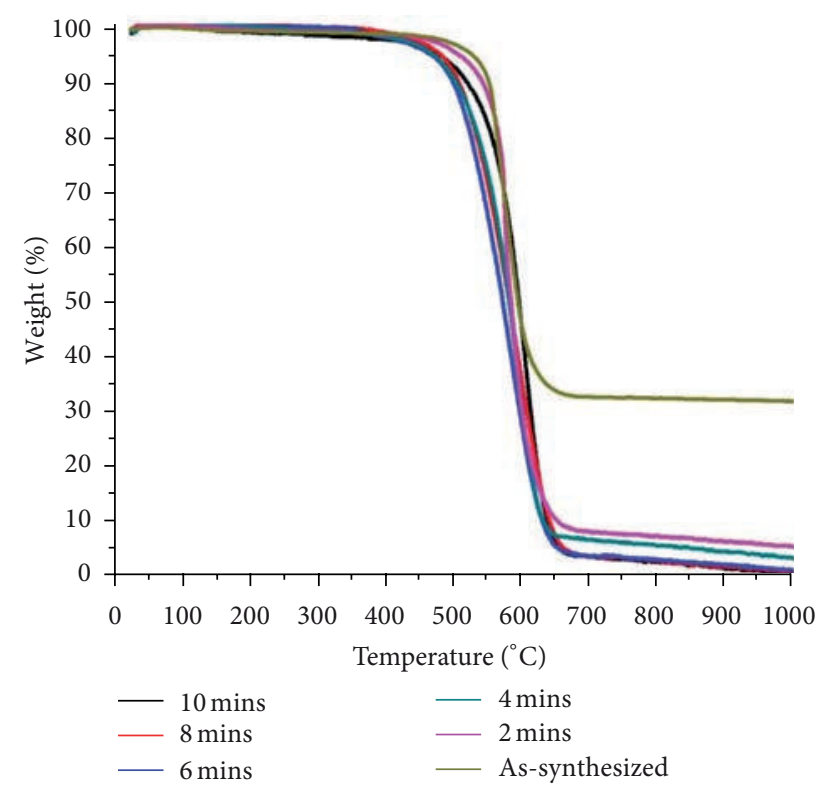

Figure 4: Graph of TG-analysis of camphoric-CNT for the assynthesized, 2, 4, 6, 8, and 10 of minutes thermal annealing time.

to $1000^{\circ} \mathrm{C}$ to evaluate thermal stability and purity of assynthesized and annealed camphoric-CNT. A plot of the weight loss monitoring thermal stability of camphoric-CNT in weight changes of the sample (including camphoric-CNT, metal catalyst, and impurities) versus temperature has been shown in Figure 4. From this diagram, it is seen that 68.01, $94.71,96.75,98.98,99.17$, and $99.75 \%$ of the total weight came from $\mathrm{C}$ element for as-synthesized, 2, 4, 6, 8, and 10 minutes of thermal annealing treatment time samples. It is found that camphoric-CNT for all samples is completely burned around $600^{\circ} \mathrm{C}$. The residual mass is the mass that was left due to orange-like powder in the sample pan at the end of the characterization process. The lowest residual iron catalyst was found to be $0.25 \%$ at 10 of minutes thermal annealing treatment time.

\section{Conclusion}

The influence of thermal annealing under nitrogen ambient on structural, micro-Raman, and TG analyses of assynthesized and annealed camphoric-CNT has been examined in to thermal annealing process; thermal annealing treatment time is the critical parameter that determines length and diameter distribution, disordered and graphitic feature, and percentage yield of camphoric-CNT. It is evaluated that the texture of camphoric-CNT changed according to the thermal annealing treatment time, by FESEM observation. Since the thermal annealing treatment time decreased the $\mathrm{I}_{D} / \mathrm{I}_{G}$ ratio of the camphoric-CNT, it also has a significant effect of graphitization degree. However, the defects in the nanotubes structure still remained but reduced in quantity; the enhancement of graphitization degree leads to a greater thermal stability that is shown in TG analysis. TG curves showed the camphoric-CNT had highest percentage yield $\sim 99.75 \%$ at 10 minutes thermal annealing treatment time. As a result, in order to produce camphoric-CNT of well-defined properties in mass production, it is advisable that further investigations required to understand the world inside nanotubes.

\section{Acknowledgments}

One of the authors (M. S. Shamsudin) is grateful to Universiti Teknologi MARA through Graduate Service Scheme and Excellence Fund (under Contact no. 600-RMI/DANA 5/3/Dst (435/2011)) for financial supports. The authors also would like to thank the Malaysian Ministry of Higher Education for providing (it is MyMaster scholarship program). Thanks are due to NANO-SciTech Centre and NANOElecTronic Centre colleagues for valuable technical assistance. The authors acknowledge the constructive comments from anonymous reviewers.

\section{References}

[1] S. Iijima, "Helical microtubules of graphitic carbon," Nature, vol. 354, no. 6348, pp. 56-58, 1991.

[2] S. Iijima and T. Ichihashi, "Single-shell carbon nanotubes of 1nm diameter," Nature, vol. 363, no. 6430, pp. 603-605, 1993.

[3] Z. Sadeghian, "Large-scale production of multi-walled carbon nanotubes by low-cost spray pyrolysis of hexane," New Carbon Materials, vol. 24, no. 1, pp. 33-38, 2009.

[4] H. Xie, A. Cai, and X. Wang, "Thermal diffusivity and conductivity of multiwalled carbon nanotube arrays," Physics Letters, Section A, vol. 369, no. 1-2, pp. 120-123, 2007.

[5] D. J. Yang, S. G. Wang, Q. Zhang, P. J. Sellin, and G. Chen, "Thermal and electrical transport in multi-walled carbon nanotubes," Physics Letters, Section A, vol. 329, no. 3, pp. 207-213, 2004.

[6] H. Rafii-Tabar, "Computational modelling of thermomechanical and transport properties of carbon nanotubes," Physics Reports, vol. 390, no. 4-5, pp. 235-452, 2004.

[7] R. B. Pipes and P. Hubert, "Helical carbon nanotube arrays: mechanical properties," Composites Science and Technology, vol. 62, no. 3, pp. 419-428, 2002. 
[8] A. Mayer and P. Lambin, "Quantum-mechanical simulations of field emission from carbon nanotubes," Carbon, vol. 40, no. 3 , pp. 429-436, 2002.

[9] A. L. Musatov, N. A. Kiselev, D. N. Zakharov et al., "Field electron emission from nanotube carbon layers grown by CVD process," Applied Surface Science, vol. 183, no. 1-2, pp. 111-119, 2001.

[10] S. L. Candelaria, Y. Shao, W. Zhou et al., "Nanostructured carbon for energy storage and conversion," Nano Energy, vol. 1, no. 2, pp. 195-220, 2012.

[11] H. Zhang and Z. Zhang, "Impact behaviour of polypropylene filled with multi-walled carbon nanotubes," European Polymer Journal, vol. 43, no. 8, pp. 3197-3207, 2007.

[12] Y. Saito and S. Uemura, "Field emission from carbon nanotubes and its application to electron sources," Carbon, vol. 38 , no. 2, pp. 169-182, 2000.

[13] C.-M. Chen, M. Chen, F.-C. Leu et al., "Purification of multiwalled carbon nanotubes by microwave digestion method," Diamond and Related Materials, vol. 13, no. 4-8, pp. 1182-1186, 2004.

[14] K. L. Strong, D. P. Anderson, K. Lafdi, and J. N. Kuhn, "Purification process for single-wall carbon nanotubes," Carbon, vol. 41, no. 8, pp. 1477-1488, 2003.

[15] D. A. Heller, P. W. Barone, and M. S. Strano, "Sonicationinduced changes in chiral distribution: a complication in the use of single-walled carbon nanotube fluorescence for determining species distribution," Carbon, vol. 43, no. 3, pp. 651-653, 2005.

[16] M. S. Shamsudin, M. F. Achoi, M. N. Asiah et al., "An investigation on the formation of carbon nanotubes by twostage chemical vapor deposition," Journal of Nanomaterials, vol. 2012, Article ID 972126, 5 pages, 2012.

[17] M. S. Shamsudin, S. Abdullah, and M. Rusop, "Structural and thermal behaviors of iron-filled align carbon nanotubes formulated by two-stage catalytic chemical vapor deposition," Advanced Materials Research, vol. 364, pp. 191-195, 2012.

[18] M. S. Shamsudin, N. A. Asli, S. Abdullah, S. Y. S. Yahya, and M. Rusop, "Effect of synthesis temperature on the growth iron-filled carbon nanotubes as evidenced by structural, microRaman, and thermogravimetric analyses," Advances in Condensed Matter Physics, vol. 2012, Article ID 420619, 7 pages, 2012.

[19] E. F. Antunes, V. G. De Resende, U. A. Mengui, J. B. M. Cunha, E. J. Corat, and M. Massi, "Analyses of residual iron in carbon nanotubes produced by camphor/ferrocene pyrolysis and purified by high temperature annealing," Applied Surface Science, vol. 257, no. 18, pp. 8038-8043, 2011.

[20] J. McKenna and H. L. Frisch, "Quantum mechanical microscopic Brownian motion," Physics Letters, vol. 19, no. 2, pp. 112-114, 1965.

[21] D. R. Tallant, J. E. Parmeter, M. P. Siegal, and R. L. Simpson, "The thermal stability of diamond-like carbon," Diamond and Related Materials, vol. 4, no. 3, pp. 191-199, 1995.

[22] Y. Ouyang and Y. Fang, "A new surface-enhanced Raman scattering system for carbon nanotubes," Spectrochimica Acta. Part A, vol. 61, no. 9, pp. 2211-2213, 2005.

[23] L. Lafi, D. Cossement, and R. Chahine, "Raman spectroscopy and nitrogen vapour adsorption for the study of structural changes during purification of single-wall carbon nanotubes," Carbon, vol. 43, no. 7, pp. 1347-1357, 2005.

[24] M. Aydin and D. L. Akins, "Calculated dependence of vibrational band frequencies of single-walled and double-walled carbon nanotubes on diameter," Vibrational Spectroscopy, vol. 53, no. 1, pp. 163-172, 2010.

[25] N. Bendiab, R. Almairac, M. Paillet, and J. L. Sauvajol, "About the profile of the tangential modes in single-wall carbon nanotube bundles," Chemical Physics Letters, vol. 372, no. 1-2, pp. 210-215, 2003.

[26] S. Kawasaki, M. Shinoda, T. Shimada, F. Okino, and H. Touhara, "Single-walled carbon nanotubes grown on natural minerals," Carbon, vol. 44, no. 11, pp. 2139-2141, 2006.

[27] N. Chiodarelli, O. Richard, H. Bender et al., "Correlation between number of walls and diameter in multiwall carbon nanotubes grown by chemical vapor deposition," Carbon, vol. 50, no. 5, pp. 1748-1752, 2012. 

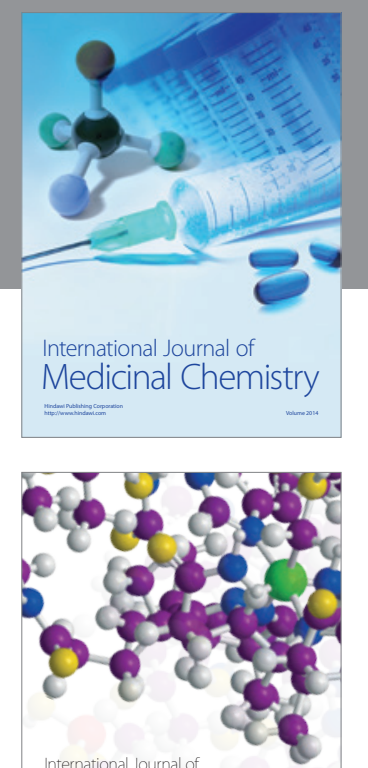

\section{Carbohydrate} Chemistry

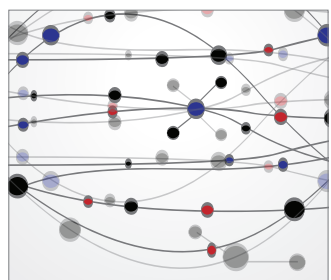

The Scientific World Journal
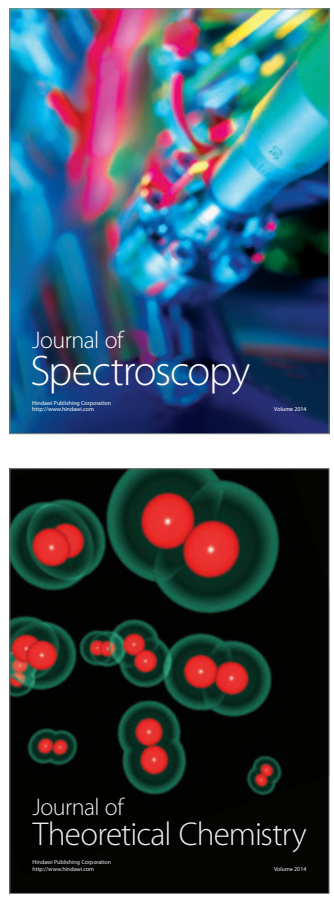
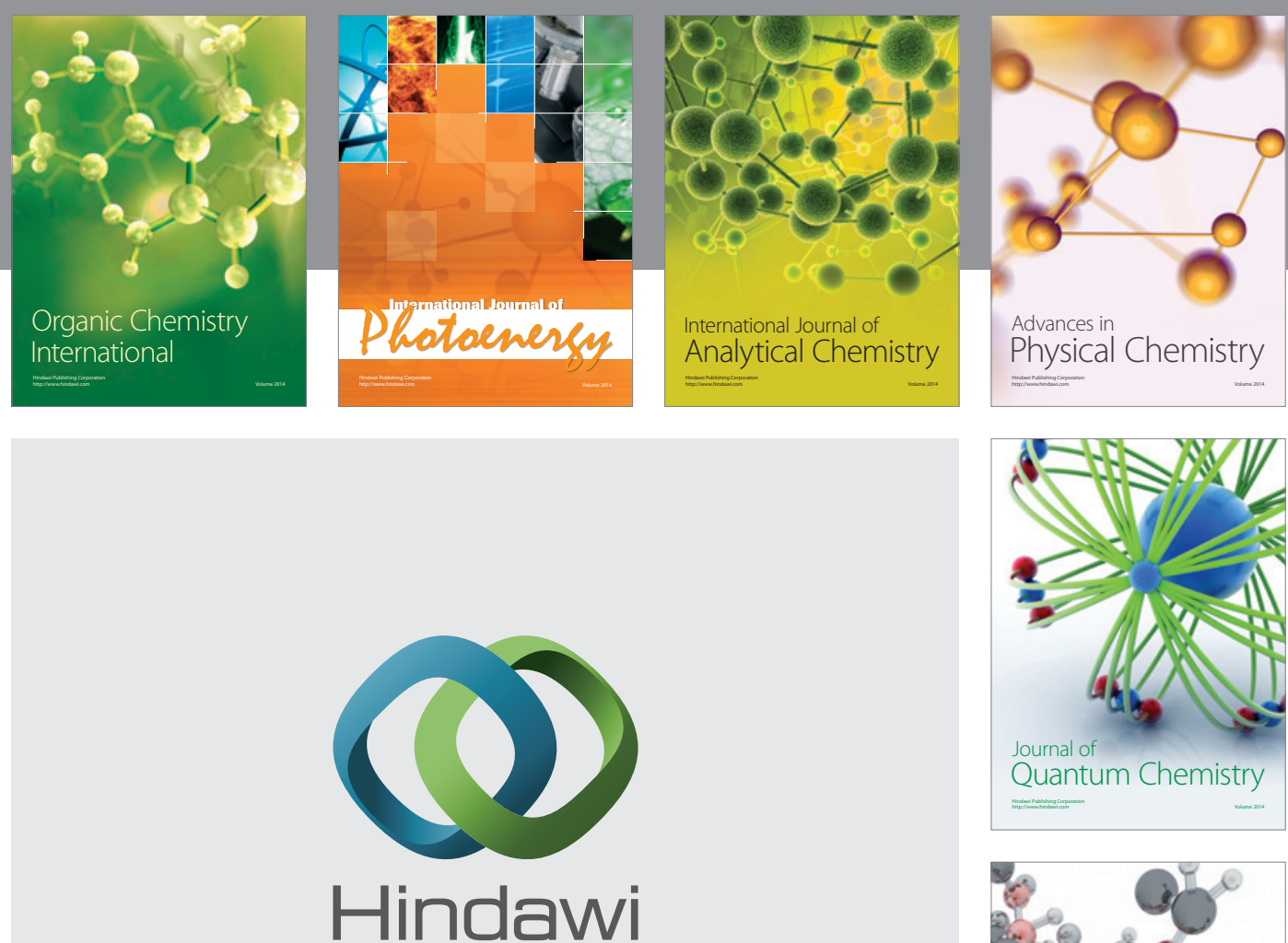

Submit your manuscripts at

http://www.hindawi.com

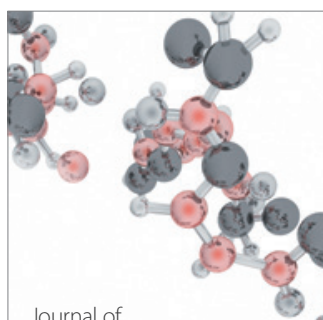

Analytical Methods

in Chemistry

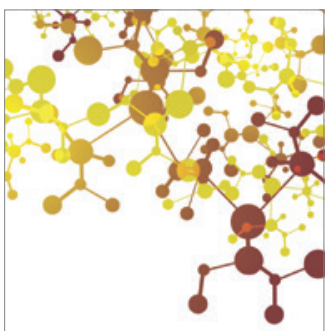

Journal of

Applied Chemistry

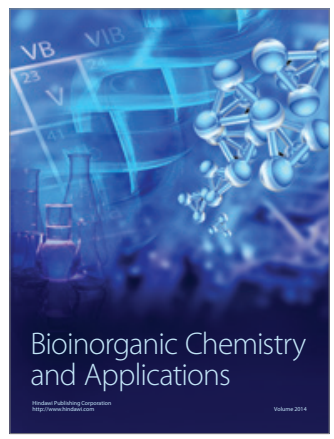

Inorganic Chemistry
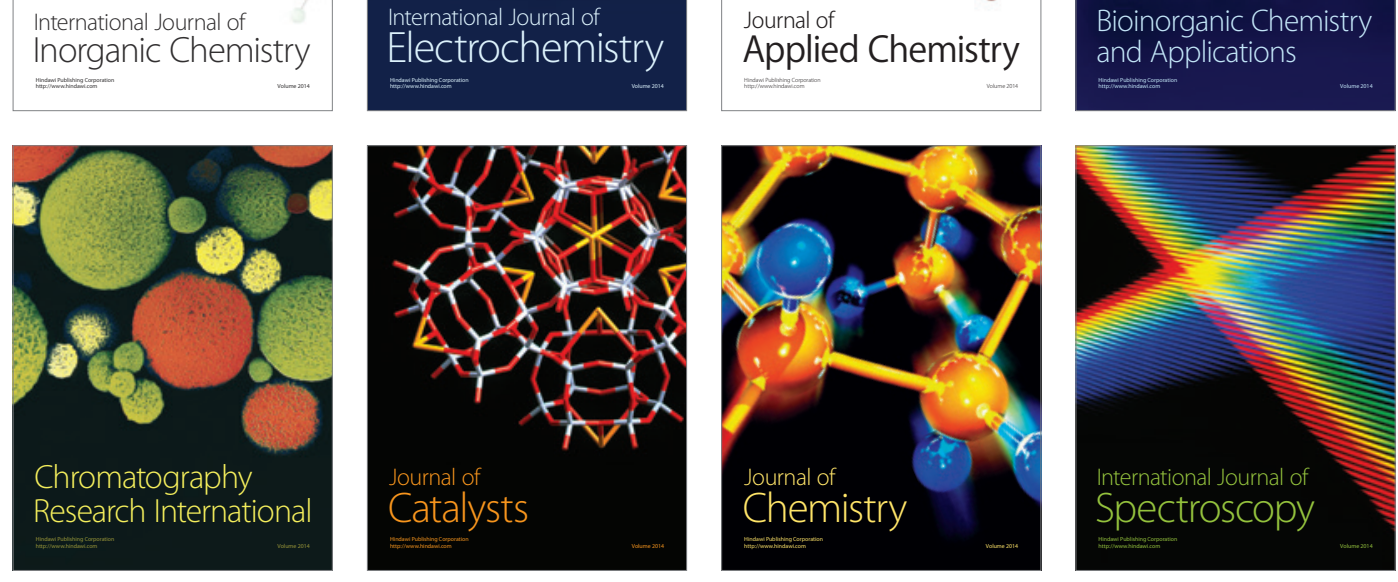